\title{
ELABORACIÓN DE FILETE SIN PIEL \\ DE PAICHE (ARAPAIMA GIGAS, CUVIER 1829) \\ AHUMADO A BAJA TEMPERATURA
}

\author{
DAVID JULIÁN ROLDÁN-ACERO \\ https://orcid.org/0000-0002-2829-6873 \\ AndrÉs Avelino MolLedA-OrdóÑEZ \\ https://orcid.org/0000-0003-2732-0752 \\ DAVID LUJÁN-TANTARICO \\ https://orcid.org/0000-0001-5077-2795 \\ JuAn ROdOLFO OMOTE-SIBINA* \\ https://orcid.org/0000-0003-1395-4973 \\ Universidad Nacional Agraria La Molina, Perú
}

Recibido: 7 de agosto del 2020 / Aprobado: 19 de agosto del 2020

doi: https://doi.org/10.26439/ing.ind2020.n039.4921

RESUMEN: El objetivo del trabajo fue elaborar un filete sin piel de paiche ahumado a baja temperatura que cumpla con estándares de calidad, inocuidad y de aceptabilidad, en los aspectos físico-químico y microbiológico. Se siguió un proceso de recepción, lavado, fileteado y cortado, inmersión en salmuera, drenado, ahumado, enfriado, envasado, congelado y almacenado. Los resultados muestran un rendimiento del filete sin piel ahumado a baja temperatura con $75,10 \%$ de humedad y $2,90 \%$ de cloruros de $35,65 \%$. Presentó $20,0 \%$ de proteína, $2,5 \%$ de grasa y $2,4 \%$ de cenizas. La energía total fue de $102,5 \mathrm{Kcal} / 100 \mathrm{~g}$ de muestra. Los resultados microbiológicos del filete ahumado se encontraron dentro de los límites que recomiendan las normas de calidad para estos productos y confirmaron las buenas condiciones sanitarias en su elaboración.

Palabras clave: ahumado en frío / paiche / salmuera

\footnotetext{
*Correo electrónico: romote@lamolina.edu.pe*, droldan@lamolina.edu.pe, amolleda@lamolina.edu.pe, davidlujan29@gmail.com
} 


\section{PRODUCTION OF COLD-SMOKED SKINLESS PAICHE (ARAPAIMA GIGAS, CUVIER 1829) FILLETS}

ABSTRACT: This work aimed to produce cold-smoked skinless paiche fillets that comply with the quality, safety and acceptability standards regarding physical, chemical and microbiological aspects. The production stages were reception, washing, slicing and cutting, brining, draining, smoking, chilling, packaging, freezing and storage. The results showed that cold-smoked skinless paiche fillets had $75.10 \%$ moisture and $35.65 \%$ of $2.90 \%$ sodium chloride. The fillets had $20.0 \%$ protein, $2.5 \%$ fat and $2.4 \%$ ash. The total energy per $100 \mathrm{~g}$ of fish was $102.5 \mathrm{kcal}$. The microbiological results of the cold-smoked fillets met the recommended quality standards for this type of product, and confirmed that good manufacturing practices were used during their production.

Keywords: cold-smoked / paiche / brine 


\section{INTRODUCCIÓN}

El paiche (Arapaima gigas, Cuvier 1829) es un recurso pesquero tradicional y popular en la cuenca del río Amazonas. Es considerado uno de los peces de agua dulce más grandes del mundo: en su medio natural puede alcanzar hasta tres metros de longitud y $250 \mathrm{~kg}$ de peso. Es una especie importante para el poblador amazónico, ya que constituye una fuente de alimentación y contribuye con la generación de ingresos a través de la comercialización de sus productos.

Alcántara y otros (2006) reportan que, a través de diversos estudios efectuados durante los últimos cinco años, tanto en Brasil como en Perú, se ha demostrado que el paiche puede ser cultivado con dietas artificiales con altos niveles de proteína. Los indicadores del cultivo de paiche en estanques y en jaulas flotantes permiten avizorar posibilidades interesantes de desarrollo en la Amazonia peruana, tanto por la gran disponibilidad de ambientes acuáticos como por la versatilidad de alimentación de la especie.

Existen muchas cartas de intención de compra de filete de paiche, tanto de Europa como de los Estados Unidos. Ello es atribuido a la alta calidad de su carne, a la ausencia de espinas y huesos intermusculares, así como al alto contenido de Omega 3, 6 y 9 . El paiche tiene una mayor preferencia en su presentación de filetes, muy similar al requerimiento del bacalao en el mercado internacional.

Según Friedrich (1989), el ahumado es un método de conservación que se utiliza para preservar productos como el pescado. El pescado ahumado está considerado un producto delicatessen por su aroma, sabor y color atractivo, para ser consumido en ocasiones especiales, o presentado como una alternativa en la dieta saludable cuando su contenido de sal es menor de $3 \%$ (Vasiliadou, 2005).

Debido a su tamaño, textura y calidad de filete, el paiche es una materia prima óptima para la industrialización a través de procesos sostenibles y sustentables que permitan su uso integral y mayores dividendos para el sector acuícola del país. Ante ello, la investigación tuvo por finalidad obtener un proceso para la elaboración de filete sin piel cortado de paiche ahumado a baja temperatura, que cumpla con las condiciones de calidad e inocuidad para alimentos de consumo humano directo.

\section{MATERIALES Y MÉTODOS}

Se utilizó paiche (Arapaima gigas) proporcionado por la empresa Silver Corporation S.A.C. ubicada en Satipo, Junín. Fue transportado en caja térmica con hielo a temperaturas $<5^{\circ} \mathrm{C}$ y posteriormente fue almacenado en el Laboratorio de Procesos Pesqueros de la UNALM. Los insumos fueron: sal de mesa y azúcar. Como fuente de humo se utilizó aserrín de madera (tornillo) y coronta seca. Se utilizó un ahumador modelo canadiense, cerradora de bolsa al vacío, congeladora doméstica Electrolux, cronómetro Casio, termómetro digital de precisión Digitron. 
Se registró la longitud total, longitud estándar y peso del paiche entero. La frescura del paiche fue evaluada sensorialmente utilizando la tabla de calidad de Wittfogel, adaptado a especies de agua dulce (Oliva, 2012). El análisis químico proximal de la materia prima y del filete sin piel cortado de paiche ahumado se realizaron utilizando los procedimientos reportados en AOAC (2012). La determinación de cloruros ( $\mathrm{NaCl}$ ) se realizó siguiendo el método NTP-ISO 1841-1:2006. Los ensayos microbiológicos del filete de paiche ahumado se realizaron según los procedimientos descritos por Ingram y otros (1983).

Las muestras experimentales fueron evaluadas sensorialmente mediante pruebas de preferencia utilizando un panel de veinte personas no entrenadas. Los formatos para la prueba de preferencia fueron elaborados según recomendación de Ureña y otros (1999) y los resultados se procesaron según el método estadístico de Friedman (Conover, 1980). El producto final fue evaluado sensorialmente utilizando un panel semientrenado de diez personas. Se utilizó el método no paramétrico de la prueba de Friedman con un nivel de significancia del $5 \%$. Los resultados obtenidos fueron procesados con el programa estadístico Minitab 17.

El diagrama de bloques de procesamiento experimental para elaborar filete sin piel cortado de paiche ahumado a baja temperatura, basado en lo reportado por Birkeland (2004), se muestra en la figura 1.

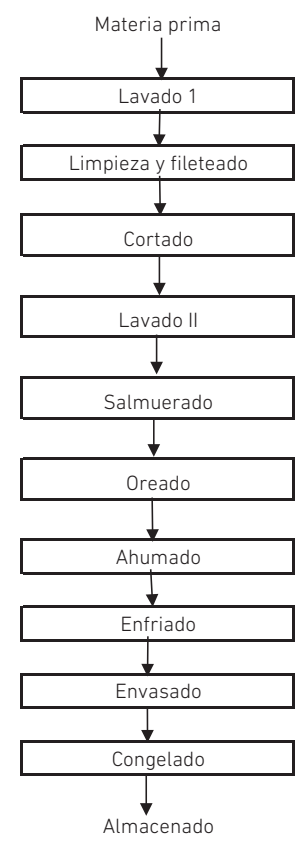

Figura 1. Diagrama de bloques para elaborar filete de paiche ahumado a baja temperatura. 
El paiche entero y fresco fue lavado, limpiado, fileteado y cortado. El tamaño de corte del filete sin piel de paiche para el ahumado a baja temperatura se determinó considerando los pesos comerciales del filete de trucha ahumada que se presentan en el mercado local de consumo masivo y que varía entre 100 y $200 \mathrm{~g}$. Se ensayaron tres pesos de filete sin piel cortado de $100 \mathrm{~g}\left(\mathrm{C}_{1}\right), 150 \mathrm{~g}\left(\mathrm{C}_{2}\right)$ y $200 \mathrm{~g}\left(\mathrm{C}_{3}\right)$. Los filetes sin piel cortados se colocaron en salmuera al $20 \%$, según recomendación de Ramírez (1978), con 10 \% de azúcar, por tiempos de inmersión de $2 \mathrm{~min}\left(\mathrm{~T}_{1}\right), 6 \mathrm{~min}\left(\mathrm{~T}_{2}\right)$ y $10 \mathrm{~min}\left(\mathrm{~T}_{3}\right)$. Posteriormente fueron escurridos al medio ambiente y oreado en el ahumador a $30{ }^{\circ} \mathrm{C}$ durante 60 a 90 minutos. El proceso de ahumado se realizó entre $30^{\circ} \mathrm{C}$ y $35^{\circ} \mathrm{C}$, siguiendo las recomendaciones de Burt (1988) y Codex Alimentarius (2020). Durante este proceso se desarrolló una curva de ahumado y una curva de pérdida de humedad. En el proceso de ahumado, se evaluaron tres tiempos de ahumado efectivo de $60 \mathrm{~min}\left(\mathrm{H}_{1}\right), 90 \mathrm{~min}\left(\mathrm{H}_{2}\right)$ y $120 \mathrm{~min}\left(\mathrm{H}_{3}\right)$. Después del ahumado, los filetes cortados se enfriaron a temperatura ambiente $\left(18^{\circ} \mathrm{C}\right.$ a $20^{\circ} \mathrm{C}$ ), envasados en bolsas de polietileno de alta densidad y posteriormente sellados al vacío. El filete sin piel cortado de paiche ahumado a baja temperatura y envasado al vacío fue llevado a congelación a $-30^{\circ} \mathrm{C}$. Finalmente se almacenó a una temperatura de $-22^{\circ} \mathrm{C}$ (Pérez, 2012).

\section{RESULTADOS}

En promedio, el paiche entero midió $111,15 \mathrm{~cm}$ de longitud total, $98,75 \mathrm{~cm}$ de longitud estándar y el peso total fue 14,105 kg. Presentó superficie brillante, de consistencia firme y elástica, con escamas fuertemente adheridas, globo ocular plano, pupila negra y córnea plateada amarillenta; las branquias presentaron coloración rojo vinoso, de mucosa clara y con los filamentos marcados. En la cavidad abdominal se encontró peritoneo liso y brillante, órganos de color rojo granate profundo y olor ligero a algas. Estas características según la tabla recomendada por Oliva (2012) dio un puntaje de 20 puntos y fue calificado de calidad extra. En la tabla 1 se observa su composición física del paiche entero.

Tabla 1

Composición física del paiche entero

\begin{tabular}{lll}
\hline Componente & Peso $(\mathrm{kg})$ & Rendimiento (\%) \\
\hline Cabeza & 1,39 & 9,87 \\
Vísceras & 0,78 & 5,51 \\
Piel & 2,39 & 16,91 \\
Restos & 2,34 & 16,61 \\
Filetes & 7,21 & 51,1 \\
Total & 14,11 & 100 \\
\hline
\end{tabular}

Elaboración propia 
El rendimiento del filete fue de 51,10\%, similar a lo reportado por Alcántara y otros (2006). La tabla 2 muestra los resultados del análisis químico del filete sin piel paiche fresco, los valores encontrados fueron muy similares a lo reportado por Vásquez y otros (2012), Pérez (2012) y Alcántara y otros (2006).

Tabla 2

Composición química proximal de filete sin piel de paiche

\begin{tabular}{lc}
\hline \multicolumn{1}{c}{ Ensayos } & Porcentaje (\%) \\
\hline Humedad $(\mathrm{g} / 100 \mathrm{~g})$ & 79,2 \\
Proteína $(\mathrm{g} / 100 \mathrm{~g}) \mathrm{F}: 6.25$ & 18,7 \\
Grasa $(\mathrm{g} / 100 \mathrm{~g})$ & 1,1 \\
Cenizas $(\mathrm{g} / 100 \mathrm{~g})$ & 1 \\
Carbohidratos $(\mathrm{g} / 100 \mathrm{~g})$ & 0 \\
Energía total $(\mathrm{kcal} / 100 \mathrm{~g})$ & 84,7 \\
\hline
\end{tabular}

\section{Elaboración propia}

El contenido de proteína del filete sin piel de paiche (18,70 \%) se encontró dentro de los rangos de $16 \%$ a $21 \%$ indicados por la FAO (Stansby, 1962; Love, 1970) para alimentos proteicos. Por su contenido de grasa (1,10 \%) fue considerado como especie magra, según reporte de Cortez (1992), para especies amazónicas.

El tamaño del filete de paiche cortado para el ahumado a baja temperatura fue definido por la prueba de preferencia entre las variables consideradas. El análisis estadístico de los resultados demostró que existían diferencias significativas $(p<0,05)$ entre las variables de peso de filete curados en salmuera al $20 \%$ y $10 \%$ de azúcar. El filete sin piel cortado de paiche con mayor preferencia fue la variable $C_{2}(150 \mathrm{~g})$ con $57 \%$ de preferencia, seguido de $\mathrm{C}_{1}(100 \mathrm{~g})$ con $24 \%$ y $\mathrm{C}_{3}(200 \mathrm{~g})$ con $19 \%$ (figura 2$)$.

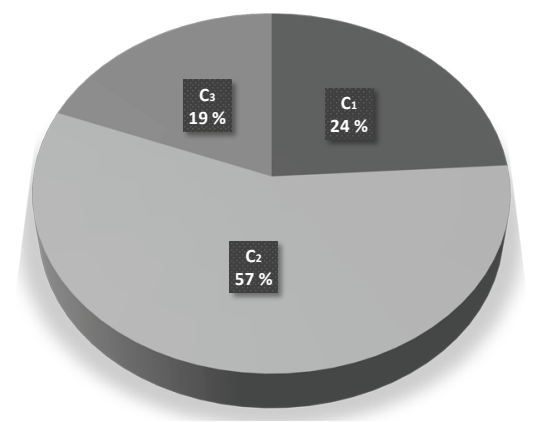

Figura 2. Preferencia de tamaño del filete sin piel cortado en función del peso

Elaboración propia 
Se realizó la curva de penetración de cloruros (figura 3) con la finalidad de medir el grado de intensidad de sabor salado en la muestra de filete de $150 \mathrm{~g}$. Se probaron los tiempos $\mathrm{T} 1=2, \mathrm{~T} 2=6$ y T3= diez minutos en una solución de salmuera al $20 \%$ con $10 \%$ de azúcar.

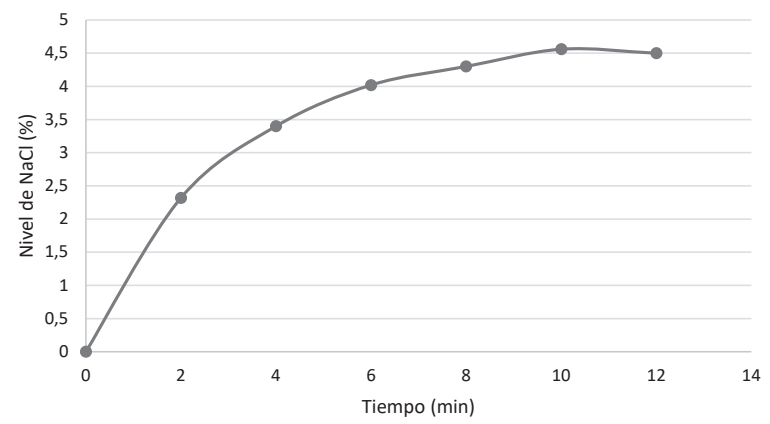

Figura 3. Curva de penetración de sal $(\mathrm{NaCl})$

\section{Elaboración propia}

La figura 3 muestra un rápido ingreso de sal $(\mathrm{NaCl})$ en el filete sin piel cortado de paiche de $150 \mathrm{~g}$ durante los primeros minutos y una tendencia constante a partir del décimo minuto. Este resultado fue similar al reportado por Chávez y otros (2012), quien, trabajando con filetes de gamitana en salmuera, reportó que el ingreso de $\mathrm{NaCl}$ en el músculo del pescado es rápido durante el primer contacto con el filete y luego se hace más constante hasta su saturación.

El filete sin piel cortado de paiche salmuerado fue ahumado y posteriormente evaluado sensorialmente por un panel, utilizando la prueba de preferencia no paramétrica de Friedman (Conover, 1980). Se utilizó una escala de 1 al 5: Excelente (5), Muy bueno (4), Bueno (3), Malo (2) y Muy malo (1). El análisis estadístico demostró que existían diferencias significativas $(p<0,05)$ entre los tratamientos estudiados. El tratamiento de mayor preferencia fue $T_{2}$ (inmersión de 6 min y 4,02 \% NaCl). Miler y Sikorski (1990) recomiendan entre $1,5 \%$ y $2 \%$ de cloruro como valores apropiados para los filetes de pescado antes del ahumado. La figura 4 muestra los resultados de la evaluación sensorial realizada en los filetes sin piel cortados de paiche ahumados. 


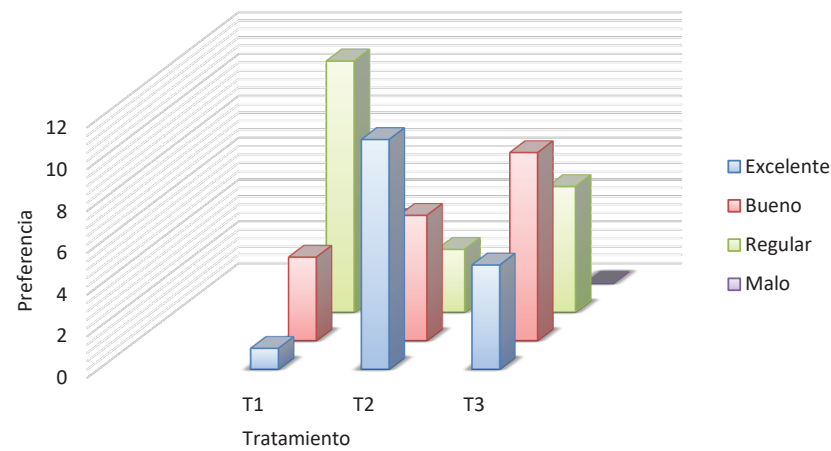

Figura 4. Preferencia de filete sin piel ahumado según el tiempo de inmersión en salmuera al $20 \%$

Elaboración propia

En el proceso de ahumado se controló la variación de temperatura. La variación de temperatura durante el proceso de ahumado se muestra en la figura 5 . El filete sin piel cortado de paiche presentó un brillo característico y agradable que fue atribuido al proceso de ahumado y azúcar utilizado. Wicki (1990) reporta que el azúcar suaviza el sabor salado del producto y le confiere una mejor coloración en la presentación.

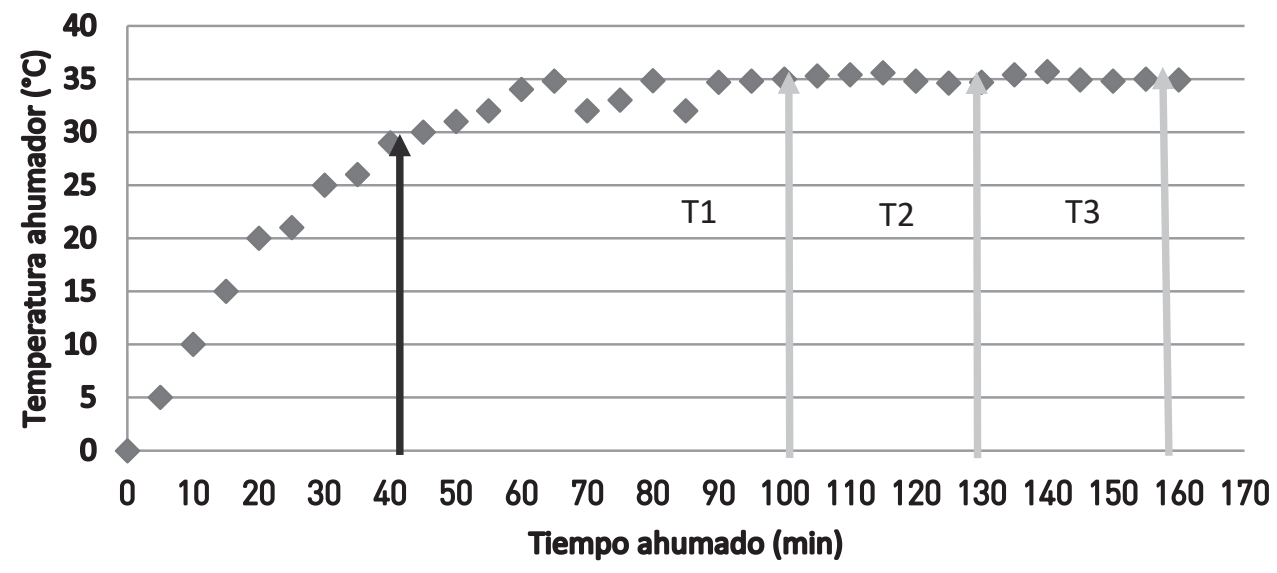

Figura 5. Variación de temperatura en proceso de ahumado de filete sin piel cortado de paiche Elaboración propia 
En la figura 5 se observa que las variaciones de la temperatura durante el proceso de ahumado se mantuvieron cercanas a los $35^{\circ} \mathrm{C}$. El tiempo de secado (oreado) del filete sin piel cortado de paiche fue de cuarenta minutos, durante los cuales se espera que el sustrato se deshidrate para que los compuestos del humo puedan adherirse con mayor facilidad. Después de este tiempo, se añadió humo en el ahumador.

El tiempo de ahumado a baja temperatura efectivo (luego del secado de $40 \mathrm{~min}$ ) fue de sesenta minutos. La temperatura de ahumado utilizada difiere del tradicional ahumado en caliente donde la temperatura puede superar los $85^{\circ} \mathrm{C}$. Roldán y Medina (2002) mencionan que el ahumado en caliente de anguila puede utilizar temperaturas mayores de $85^{\circ} \mathrm{C}$ y durar entre 2,5 y 3,5 horas. Kenneth y Hildebrant (1996) reportan que las primeras horas de proceso de ahumado son una etapa controlada, con poca variación de temperatura, tal como se observa en la curva de temperatura interna reportada para el proceso de ahumado a baja temperatura.

La pérdida de peso del filete sin piel cortado de paiche (figura 6) durante el proceso de ahumado a baja temperatura fue baja (6,3\% al final de proceso); se utilizó una muestra de menor peso como referencia en paralelo $(70-80 \mathrm{~g})$ y se notó la misma tendencia, tal como se observa en la figura 8. Esta pérdida de peso fue atribuida al poco tiempo de secado y ahumado efectivo realizado, ya que no generó una elevada pérdida de humedad en las muestras ensayadas. La pérdida de peso por pérdida de humedad es atribuida al proceso de secado y ahumado. Wignall (1991) menciona que, para filetes de anguila ahumada en caliente que se trabaja en Europa, la pérdida normal está alrededor a $20 \%$.

La evaluación sensorial de las muestras del filete sin piel cortado de paiche ahumado a baja temperatura en diferentes tiempos de ahumado se realizó con un panel semientrenado de diez personas.

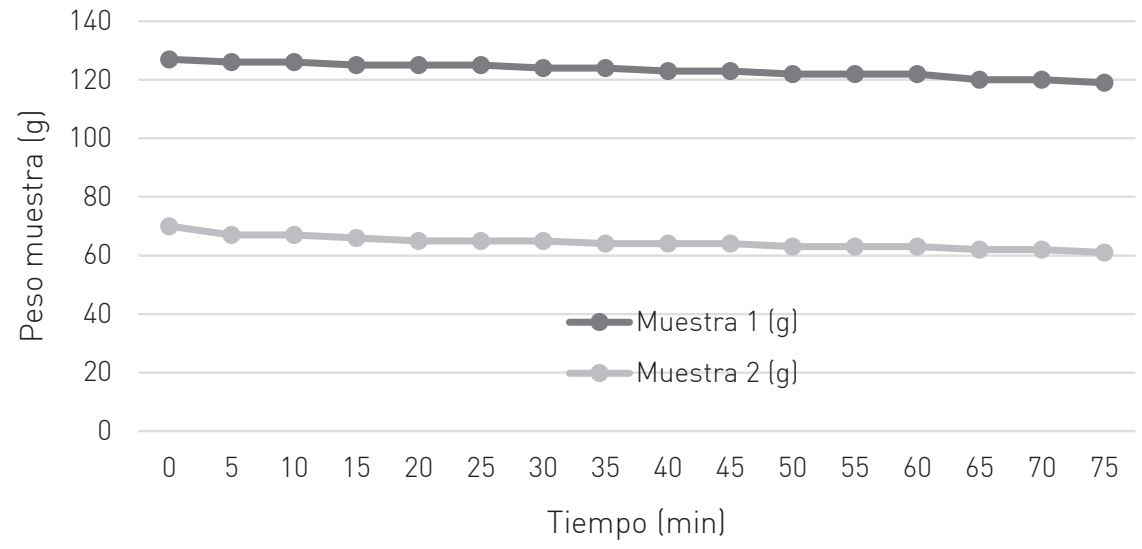

Figura 6. Control de la pérdida de peso del filete sin piel cortado durante el ahumado 
El análisis estadístico de los resultados obtenidos demostró que existen diferencias significativas entre los tratamientos estudiados y que el tratamiento de mayor preferencia fue el tratamiento $\mathrm{H}_{1}$ (ahumado por $1 \mathrm{~h}$ ). La muestra de este tratamiento presentó un mejor balance entre las características de humo y el sabor del filete, lo que fue atribuido a que el ahumado a baja temperatura permitió resaltar y conservar las propiedades organolépticas del filete cortado procesado (figura 7). Al respecto, Durruty y Corti (2013) reportan que el efecto del ahumado en el alimento genera modificaciones de las características sensoriales en el aspecto, aroma y el sabor a ahumado. Esto no solo depende de los componentes del humo, sino también de las reacciones con el sustrato; en este caso, del filete de paiche.

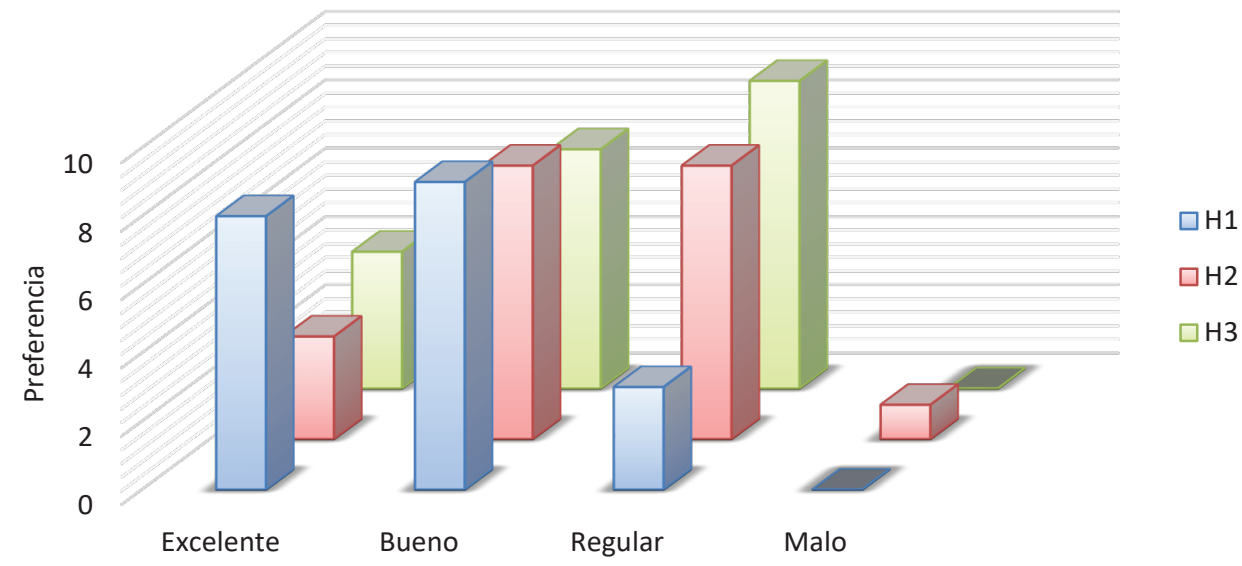

Figura 7. Preferencia del producto ahumado en función del tiempo

Elaboración propia

La aceptabilidad del producto ahumado de paiche se realizó mediante un panel semientrenado de diez personas, utilizando cinco descriptores de calidad: sabor, aspecto, olor, textura y condiciones del vacío. Al aplicar la prueba de Friedman, se obtuvo que la evaluación realizada al producto sellado al vacío no tuvo significancia $(p>0,05)$ con respecto a los demás descriptores evaluados (tabla 3). Los valores promedio para cada descriptor indicaron que su aceptabilidad es mejor calificada en cuanto al olor, textura y sabor. 
Tabla 3

Prueba de evaluación sensorial de filetes de paiche ahumado

\begin{tabular}{lllll}
\hline Atributo de calidad & & \multicolumn{1}{c}{ Tratamiento } & H2 & H3 \\
& & $\mathrm{H} 1$ & $4,17 \pm 0,52$ & $*$ \\
\hline Aspecto & $\mathrm{L}$ & $4,50 \pm 0,61$ & $3,33 \pm 0,39$ & $*$ \\
Olor & $4,67 \pm 0,83$ & $4,00 \pm 0,60$ & $4,00 \pm 0,44$ & $*$ \\
Sabor & $5,00 \pm 0,76$ & $5,00 \pm 0,61$ & $4,17 \pm 0,43$ & $*$ \\
Textura & $4,83 \pm 0,78$ & $4,50 \pm 0,59$ & $5,00 \pm 0,61$ & n.s. \\
Vacío & $5,00 \pm 0,61$ & $5,00 \pm 0,59$ & & \\
\hline
\end{tabular}

Elaboración propia

La tabla 4 muestra los resultados del análisis químico del filete sin piel cortado de paiche ahumado a baja temperatura. Los resultados de humedad y proteína fueron muy cercanos a lo reportado por Pérez (2012), quien evaluó filetes de paiche ahumado. Ello puede ser atribuido al corto tiempo de secado durante el ahumado, que disminuye el contenido de humedad y permite el aumento el contenido de proteína. Asimismo, el contenido de grasa fue casi el $50 \%$ menos. Este menor contenido puede deberse a las diferentes condiciones de crianza, estado de crecimiento o estación de año que afectan el contenido graso en los filetes de paiche (Salas y Barriga, 2004).

Tabla 4

Composición química del filete sin piel cortado de paiche ahumado a baja temperatura

\begin{tabular}{lr}
\hline \multicolumn{1}{c}{ Ensayos } & \multicolumn{1}{c}{$\%$} \\
\hline Humedad (g/100 g muestra) & 75,10 \\
Proteína (g/100 g muestra) Factor: 6,25 & 20,00 \\
Grasa (g/100 g muestra) & 2,50 \\
Cenizas (g/100 g muestra) & 2,40 \\
Carbohidratos (g/100 g muestra) & 0,00 \\
Energía total (kcal/100 g muestra) & 102,50 \\
\hline
\end{tabular}

Elaboración propia

Fernández y otros (1995) reportaron que el proceso de ahumado afecta al valor nutritivo del pescado principalmente por la reducción de la biodisponibilidad de las proteínas. Sin embargo, considerando que el proceso de ahumado a baja temperatura (próximo a los $35^{\circ} \mathrm{C}$ ) no implica temperaturas lo suficientemente altas para reducir su valor biológico, simplemente se generan enlaces cruzados o pardeamiento inducidos por el calor, con pérdidas relativamente pequeñas en aminoácidos y, por ende, de proteínas. 
Por otro lado, el poco tiempo de exposición al humo permite asegurar que se acentúen las características sensoriales de producto ahumado atribuido a la deposición de fase niebla del humo considerada de baja o nula toxicidad.

Daun (1979) reporta que el uso actual del humo es principalmente como agente saborizante y sus efectos benéficos incluyen la formación de color y sabor característicos. Asimismo, indica que el desarrollo del color es causado principalmente por la acción de los carbonilos en la fase de vapor del humo con los grupos amino primarios en la superficie del alimento como producto de una serie de reacciones enzimáticas similares a la reacción de Maillard.

Los resultados del análisis microbiológico del filete sin piel cortado de paiche ahumado se reportan en la tabla 5. Comparando los resultados microbiológicos con los límites máximos permisibles recomendados por DIGESA (2003), el filete sin piel cortado de paiche ahumado se encuentra libre de microorganismos dañinos para la salud humana.

Tabla 5

Análisis microbiológico de filete sin piel cortado de paiche ahumado a baja temperatura

\begin{tabular}{lc}
\hline \multicolumn{1}{c}{ Ensayos } & Recuento \\
\hline N. aerobios mesófilos viables (UFC/g) & 10 \\
N. Staphylococcus aureus (NMP/g) & $<3$ \\
N. Clostridium perfringens (UFC/g) & $<10$ \\
\hline
\end{tabular}

Elaboración propia

Los valores reportados en el análisis microbiológico y el estado congelado del filete sin piel cortado de paiche ahumado a baja temperatura permitirían un adecuado período de vida útil del producto. Esta vida útil es variable debido a las distintas posibilidades de combinación de factores de conservación utilizados y al grado de deshidratación que presentan, el efecto combinado de la sal, de los constituyentes del humo y del secado que acompaña al proceso de ahumado (Fernández et al., 1995).

El rendimiento del filete sin piel cortado de paiche ahumado a baja temperatura se muestra en la tabla 6 y fue realizado en función del peso de la materia prima entera. 
Tabla 6

Rendimiento de filete sin piel cortado de paiche ahumado a baja temperatura

\begin{tabular}{lcc}
\hline Componente & Peso (g) & Rendimiento (\%) \\
\hline Paiche entero & 14,11 & 100,00 \\
Filete sin piel de paiche & 7,20 & 51,10 \\
Filete sin piel cortado de paiche & 5,37 & 38,05 \\
Recortes de filete sin piel & 1,83 & 12,96 \\
Filete sin piel ahumado de paiche & 5,03 & 35,65 \\
\hline
\end{tabular}

Elaboración propia

El resultado del rendimiento del filete sin piel cortado de paiche ahumado a baja temperatura $(35,65 \%)$ fue mayor que el rendimiento promedio $(28 \%)$ reportado por IIAP (2000), para filete con piel ahumado para peces de cautiverio (paco, gamitana y boquichico). Esta diferencia puede ser atribuida a la diferente presentación y a que el secado y ahumado efectivo se realizó en poco tiempo.

\section{CONCLUSIONES}

El proceso para elaborar filete sin piel cortado de paiche (Arapaima gigas, Cuvier, 1829) ahumado a baja temperatura fue: recepción - Lavado 1 - Limpiado y fileteado - Cortado - Lavado 2 - Salmuerado (20 \% sal y $10 \%$ azúcar) - Oreado (60-90 min) - Ahumado a baja temperatura $\left(35^{\circ} \mathrm{C} \times 1 \mathrm{~h}\right)$ - Enfriado - Envasado - Congelado $\left(-30^{\circ} \mathrm{C}\right)$ - Almacenado $\left(-22^{\circ} \mathrm{C}\right)$.

- El filete sin piel cortado de paiche de mayor aceptación fue el de 120-130 g

- El tiempo de inmersión del filete sin piel cortado de 120-130 g en salmuera de $20 \%$ con $10 \%$ de azúcar fue de seis minutos.

- La concentración de cloruros de mayor preferencia fue la de 4,02 \%.

- El tiempo de ahumado efectivo del filete sin piel cortado de paiche a $35{ }^{\circ} \mathrm{C}$ fue de una hora.

- La composición química del filete sin piel cortado de paiche ahumado a baja temperatura fue: humedad $75,10 \%$, proteína $20,00 \%$, grasa $2,50 \%$, cenizas $2,4 \%$ y energía total $102,5 \mathrm{kcal} / 100 \mathrm{~g}$ muestra.

- El rendimiento del filete sin piel cortado de paiche ahumados a baja temperatura fue de $35,65 \%$ a partir de la materia prima entera. 


\section{REFERENCIAS}

Alcántara, F.; Wust, W.; Rebaza, M. y Del Castillo, A. (2006). Paiche, el gigante del Amazonas. Instituto de Investigaciones de la Amazonia Peruana. Recuperado de http://www. iiap.org.pe/Upload/Publicacion/L031.pdf

AOAC (2012). Official Methods of Analysis, Association of Official Analytical Chemist Washington, D. C.: Association of Official Agricultural Chemist.

Birkeland, S. (2004). Effects of cold smoking procedures and raw material characteristics on product yield and quality parameters of cold smoked Atlantic salmon (Salmo salar L.) fillets. Food Research International, 37, 273-286.

Burt, J.R. (1988). Fish smoking and drying: the effect of smoking and drying on the nutritional properties of fish. London: Elsevier Applied Science.

Chávez, T.; Araujo, R. y Bernuy, J. (2012). Technical study for the elaboration of cured products from Colossoma macropomum (gamitana) using the technique of dry-salted in wet and dry cell battery. Conocimiento Amazónico, 3(1), 3-16.

Codex Alimentarius (21 de mayo del 2020). Principles and application of the HACCP system.

Conover, W. J. (1980). Practical Nonparametric Statistics. New York: John Wiley \& Sons.

Cortez, J. (1992). Características bromatológicas de dieciséis especies hidrobiológicas de la Amazonia peruana en época de creciente. Folia Amazónica, 4, 115-122.

Daun, H. (1979). Interaction of wood smoke components and foods. Food Technology, 2 , 66-70.

DIGESA (2003). Criterios microbiológicos de calidad sanitaria e inocuidad para los alimentos y bebidas de consumo humano. Normas legales.

Durruty, M. y Corti, I. (2013). Análisis físico-químico, sensorial y consumo de productos pesqueros ahumados. Mar del Plata: Departamento de Metodología de la Investigación de la Universidad FASTA.

Fernández, S.; Pollak, A. y Vitancurt, J. (1995). Pescado ahumado artesanalmente - Ensayos tecnológicos. Montevideo: Programa de Conservación de la Biodiversidad y Desarrollo Sustentable los Humedales del Este (PROBIDES).

IIAP (2000). Instituto de Investigaciones de la Amazonía Peruana. Recuperado de http:// www.iiap.org.pe/Upload/Publicacion/CultivProcesPeces.pdf

Ingram, M.; Bray, D.; Clark, D.; Dolman, C.; Elliot, R. y Thetcher, F. (1983). Microorganismos de los alimentos. Técnicas de análisis microbiológicos. Zaragoza: Acribia S. A.

Kenneth, S. y Hildebrand, J. R. (1996). Smoking Fish at Home Safely. Ciudad: Pacific Northwest Extension Publication PNW. 
Love, R. M. (1970). The Chemical Biology of Fishes. London: Academic Press.

Miler, K. y Sikorski, Z. (1990). Tecnología de los productos del Mar. Recursos, composición nutritiva y conservación. Zaragoza: Acribia.

NTP-ISO 1841-1:2006 (18 de mayo del 2020). Norma Técnica Peruana. Recuperado de https://www.deperu.com/normas-tecnicas/NTP-ISO-1841-1.html

Oliva, G. (2012). Elaboración de conservas de tilapia (Orechronus miloticus) en salsas de sachatomate (Solanum betacea Cav.) [tesis para optar el título de ingeniero pesquero, Universidad Nacional Agraria La Molina, Perú].

Pérez, R. (2012). Procesamiento y productos con valor agregado del paiche (Arapaima gigas). Callao: Dirección de Transferencia Tecnológica y Desarrollo para el Consumo (DTTDC).

Roldán, D. y Medina, C. (2002). Evaluación del comportamiento de filetes de anguila común en el procesamiento de ahumado en caliente. Anales científicos, LIII, 489-505.

Salas, A. y Barriga, M. (2004). Aspectos bioquímicos y cambios post mortem del filete de paiche (Arapaima gigas) almacenado en hielo. Boletín de investigación del Instituto Pesquero del Perú, 6, 27-32.

Stansby, M. E. (1962). Fish in nutrition. London: Fishing News Books Ltd.

Ureña, M.; D’arriego, M. y Girón, O. (1999). Evaluación sensorial de los alimentos, aplicación didáctica. Lima: Ediagraria.

Vasiliadou, S. (2005). Effect of smoking on quality parameters of farmed gilthed sea bream (Sparus aurata L.) and sensory attributes of the smoked product. Eur Food Res Technology, 221(3-4), 232-236.

Vásquez, D.; Córdova, C.; Olórtegui, W.; Cachique, N.; Silva, L. y García, R. (2012), Valor agregado de las especies: Brycon erythropterum (sábalo), Colossoma macropomum (gamitana), Arapaima gigas (paiche) y Agouti paca (majas). Ciencia Amazónica, 2(2), 135-141.

Wicki, G. A. (1990). El proceso de ahumado como valor agregado en la producción del Catfish Sudamericano (Rhamdia sapo). Recuperado de https://docplayer. es/6675262-El-proceso-de-ahumado-como-valor-agregado-en-la-producciondel-catfish-sudamericano-rhamdia-sapo-gustavo-a-wicki-introduccion.html

Wignall, J. (1991). Curso Internacional de Tecnología de Productos Pesqueros: El ahumado de pescado. Santiago de Chile: Fundación Chile. 